https://doi.org/10.15407/ujpe66.2.105

M. AYGUN

Department of Physics, Bitlis Eren University

(Bitlis, Turkey; e-mail: murata.25@gmail.com)

\title{
INFLUENCE OF DEFORMED SURFACE DIFFUSENESS ON ELASTIC SCATTERING REACTIONS INVOLVING ACTINIDE AND LANTHANIDE TARGETS
}

\begin{abstract}
The effect of the deformed surface diffuseness on the elastic scattering reactions with actinide and lanthanide targets is examined. The elastic scattering cross-sections are calculated by assuming the spherical structure for the projectiles and both spherical and deformed structures for the target nuclei. The theoretical calculations are performed by using spherical and deformed Broglie-Winther potentials for the real potential and the Woods-Saxon potential for the imaginary potential in the framework of the optical model. Finally, the effect of the angle dependence on the deformed surface diffuseness for two different orientation angles such as $\theta=\pi / 4$ and $\theta=\pi / 2$ is studied. All the theoretical results are compared with both one another and experimental data.
\end{abstract}

Ke ywords: nuclear surface diffuseness, deformation and orientation, elastic scattering, optical model.

\section{Introduction}

The elastic scattering reaction has a great importance in nuclear physics. It provides important information about nuclear structural and nucleus interactions. Nuclear potentials play a critical role in the analysis of elastic scattering reactions. If the nuclear potential defining the system is adequately identified, then the interaction can be well defined. For this purpose, different nuclear potentials such as the WoodsSaxon (WS) potential [1], proximity potentials [2], and double folding potential [3] are available from the literature. Although these potentials are applied to elastic scattering and fusion reactions [4-6], the application of proximity potentials to elastic scattering reactions has recently been expanded [7-11].

The Broglie-Winther (BW91) potential is one of the most used proximity potentials. It is formulated basing on the Woods-Saxon parametrization. In addition, the surface diffusion parameter is one of the effective parameters in defining the potential. The effect of this parameter has been examined for fusion reactions $[12,13]$ and the alpha decay half-lives [14], but not for the analysis of elastic scattering reactions. Therefore, we think that it will be a new and interesting study to investigate the effect of a de-

(c) M. AYGUN, 2021

ISSN 2071-0194. Ukr. J. Phys. 2021. Vol. 66, No. 2 formed surface diffusion parameter on the elastic scattering reactions with both actinide and lanthanide targets.

In the present work, we examine the effect of a deformed surface diffuseness on the elastic scattering reactions. For this, we calculate the elastic scattering cross-sections for the actinide $\left({ }^{6} \mathrm{Li}+{ }^{232} \mathrm{Th}\right.$, ${ }^{6} \mathrm{Li}+{ }^{238} \mathrm{U},{ }^{7} \mathrm{Li}+{ }^{232} \mathrm{Th}$ and ${ }^{7} \mathrm{Li}+{ }^{238} \mathrm{U}$ ) and lanthanide $\left({ }^{12} \mathrm{C}+{ }^{152} \mathrm{Sm},{ }^{14} \mathrm{~N}+{ }^{159} \mathrm{~Tb},{ }^{18} \mathrm{O}+{ }^{174} \mathrm{Yb}\right.$ and $\left.{ }^{19} \mathrm{~F}+{ }^{159} \mathrm{~Tb}\right)$ target reactions by using the deformed surface diffuseness parameter. Then, we investigate the effect of the angle dependence on the deformed surface diffuseness for two different orientation angles such as $\theta=\pi / 4$ and $\theta=\pi / 2$. Finally, we compare all the theoretical results with the experimental data and make appropriate comments on them.

The present paper is organized as follows. In Section 2 , we briefly give the calculation procedure used for the theoretical analysis. In Section 3, the results and discussion are provided. In Section 4, the summary and conclusions of the work are given.

\section{Theoretical Calculation Procedure}

The effective interaction potential for a spherical projectile and a deformed target nucleus can be written as

$U(r)=V_{\mathrm{C}}(r)+V(r)+i W(r)$, 
where $V_{\mathrm{C}}$ is the Coulomb potential, $V(r)$ is the real potential, and $W(r)$ is the imaginary potential. The $V_{\mathrm{C}}$ potential is taken as [15]

$V_{\mathrm{C}}(r)=\frac{1}{4 \pi \epsilon_{\circ}} \frac{Z_{P} Z_{T} e^{2}}{r}, \quad r \geq R_{\mathrm{C}}$,

$\frac{1}{4 \pi \epsilon_{\circ}} \frac{Z_{P} Z_{T} e^{2}}{2 R_{\mathrm{C}}}\left(3-\frac{r^{2}}{R_{\mathrm{C}}^{2}}\right), \quad r<R_{\mathrm{C}}$

$R_{\mathrm{C}}=1.25\left(A_{P}^{1 / 3}+A_{T}^{1 / 3}\right)$.

The $V(r)$ potential is evaluated as the both spherical Broglie-Winther (BW91) potential and deformed Broglie-Winther (DBW91) potential. These potentials are defined in the following subsections. Finally, the $W(r)$ potential is given by

$W(r)=-\frac{W_{0}}{1+\exp \left(\frac{r-r_{w}\left(A_{P}^{1 / 3}+A_{T}^{1 / 3}\right)}{a_{w}}\right)}$,

where $W_{0}$ is the depth, $r_{w}$ is the radius, and $a_{w}$ is the diffuseness parameter. The elastic cross-section calculations are performed by using the FRESCO code [16].

\subsection{Spherical Broglie-Winther (BW91) potential}

The spherical Broglie-Winther (BW91) potential [17] is used as [18]

$V_{N}^{\mathrm{BW} 91}(r)=-\frac{V_{0}}{\left[1+\exp \left(\frac{r-R_{0}}{a}\right)\right]} \mathrm{MeV}$,

where

$V_{0}=16 \pi \frac{R_{p} R_{t}}{R_{p}+R_{t}} \gamma a, \quad a=0.63 \mathrm{fm}$,

and

$$
\begin{aligned}
& R_{0}=R_{p}+R_{t}+0.29 \\
& R_{p, t}=1.233 A_{p, t}^{1 / 3}-0.98 A_{p, t}^{-1 / 3}
\end{aligned}
$$

with

$\gamma=0.95\left[1-1.8\left(\frac{N_{p}-Z_{p}}{A_{p}}\right)\left(\frac{N_{t}-Z_{t}}{A_{t}}\right)\right]$.

\subsection{Deformed Broglie-Winther (DBW91) potential}

The deformed Broglie-Winther (DBW91) potential is assumed in the following form

$V_{N}^{\mathrm{DBW} 91}(r, \theta)=-\frac{V_{0}(\theta)}{\left[1+\exp \left(\frac{r-R_{0}(\theta)}{a(\theta)}\right)\right]} \mathrm{MeV}$,

where

$V_{0}(\theta)=16 \pi \frac{R_{p} R_{t}(\theta)}{R_{p}+R_{t}(\theta)} \gamma a(\theta)$.

The average formula of the deformed surface diffuseness parameters $a(\theta)$ for actinides at $0 \leq \theta \leq \pi / 2$ is accepted as

$a(\theta)=a(0)+0.45 \sin ^{2} \theta-0.4, \sin ^{3} \theta$,

and, for rare-earth nuclei,

$a(\theta)=a(0)+0.25 \sin ^{2} \theta-0.25 \sin ^{3} \theta$.

The nuclear interaction potential radius $R_{0}(\theta)$ is given by

$R_{0}(\theta)=R_{p}+R_{t}(\theta)+0.29$,

where

$R_{i}(\theta)=\left(1.233 A_{i}^{1 / 3}-0.98 A_{i}^{-1 / 3}\right) \times$

$\times\left[1+\beta_{2 i} Y_{20}(\theta)+\beta_{4 i} Y_{40}(\theta)\right] \quad(i=p, t)$.

The $\boldsymbol{\beta}_{2}$ and $\boldsymbol{\beta}_{4}$ show the quadrupole and hexadecapole deformation parameters of deformed targets, respectively. The $\beta_{2}$ and $\beta_{4}$ values of the targets investigated in this study are listed in Table 1.

Table 1. The $\boldsymbol{\beta}_{\mathbf{2}}$ and $\boldsymbol{\beta}_{\mathbf{4}}$ values

of actinide and lanthanide target nuclei

examined in our study [19]

\begin{tabular}{|l|r|r|}
\hline Target & $\beta_{2}$ & $\beta_{4}$ \\
\hline${ }^{152} \mathrm{Sm}$ & 0.237 & 0.097 \\
${ }^{159} \mathrm{~Tb}$ & 0.271 & 0.066 \\
${ }^{174} \mathrm{Yb}$ & 0.289 & -0.042 \\
${ }^{232} \mathrm{Th}$ & 0.205 & 0.103 \\
${ }^{238} \mathrm{U}$ & 0.236 & 0.098 \\
\hline
\end{tabular}

ISSN 2071-0194. Ukr. J. Phys. 2021. Vol. 66, No. 2 


\section{Results and Discussion}

In our study, the effects of deformations and orientations on the elastic scattering cross-sections have been investigated within the framework of the optical model. For this, the target nuclei have been assumed as deformed nuclei at different orientations, while the projectiles have been taken as the spherical nuclei.

In Fig. 1, we have plotted the real parts of nuclear potentials as functions of the distance $(r)$ for spherical targets of actinides and lanthanides and those with deformed surface diffuseness. The real potentials have been acquired by using the FORTRAN code which is written by us. It has been seen that the tailing of the potentials of both actinide and lanthanide nuclei decreases with increasing the orientation angle. For the real potential depths of the reactions with actinide and lanthanide targets, it has been observed that the potentials at $\theta=\pi / 4$ are deeper than the potentials at $\theta=\pi / 2$.

The investigation of elastic scattering reactions with deformed surface diffuseness has been firstly performed for actinide targets. For this purpose, the elastic scattering angular distributions of ${ }^{6} \mathrm{Li}+{ }^{232} \mathrm{Th}$, ${ }^{6} \mathrm{Li}+{ }^{238} \mathrm{U},{ }^{7} \mathrm{Li}+{ }^{232} \mathrm{Th}$ and ${ }^{7} \mathrm{Li}+{ }^{238} \mathrm{U}$ reactions available from the literature have been calculated for both spherical and deformed surface diffuseness cases at two different orientation angles. In addition to this, $\chi^{2} / N$ values for each reaction and situation have been calculated and given in Table 2 . The theoretical results have been shown as compared with the experimental data in Fig. 2. It has been seen that the results of the spherical and $\theta=\pi / 4$ situations of ${ }^{6} \mathrm{Li}+{ }^{232} \mathrm{Th}$ and ${ }^{6} \mathrm{Li}+{ }^{238} \mathrm{U}$ reactions are in good agreement with each other and the experimental data. For ${ }^{7} \mathrm{Li}+{ }^{232} \mathrm{Th}$ reaction, the results of the spherical and $\theta=\pi / 2$ show very similar behavior with each other. However, the results of $\theta=\pi / 4$ are in better agreement with the experimental data than the results of the spherical and $\theta=\pi / 2$. For ${ }^{7} \mathrm{Li}+{ }^{238} \mathrm{U}$ reaction, although the results of the spherical and $\theta=\pi / 4$ at small angles show similar behavior with each other, this situation occurs between the results of spherical and $\theta=\pi / 2$ at forward angles. In addition, the results of the spherical and $\theta=\pi / 4$ are better than the results of the $\theta=\pi / 2$.

Then, the examination of the elastic scattering reactions with deformed surface diffuseness has been carried out for ${ }^{12} \mathrm{C}+{ }^{152} \mathrm{Sm},{ }^{14} \mathrm{~N}+{ }^{159} \mathrm{~Tb}$,

ISSN 2071-0194. Ukr. J. Phys. 2021. Vol. 66, No. 2

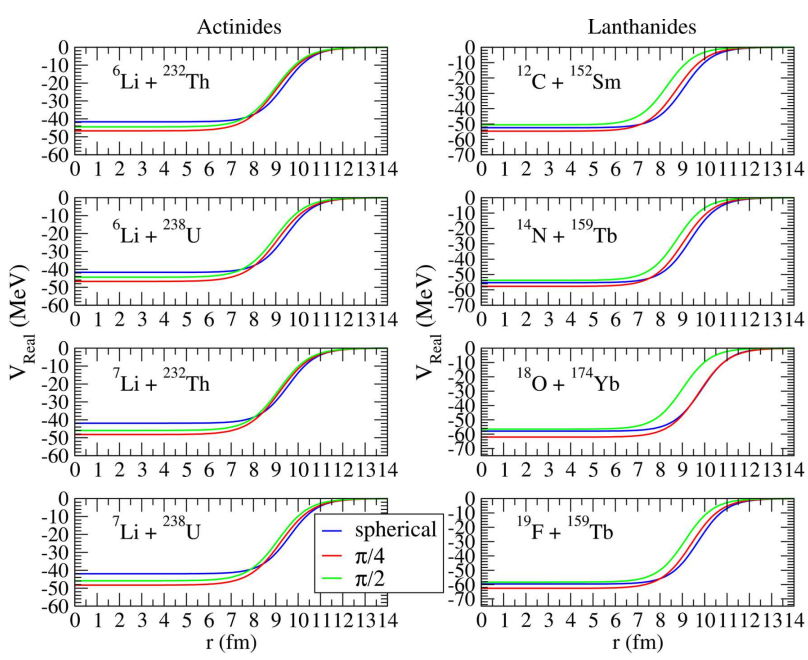

Fig. 1. Distance-dependent changes of the real parts of nuclear potentials for spherical diffuseness parameter, deformed diffuseness parameter, and different orientations for the actinide and lanthanide target reactions
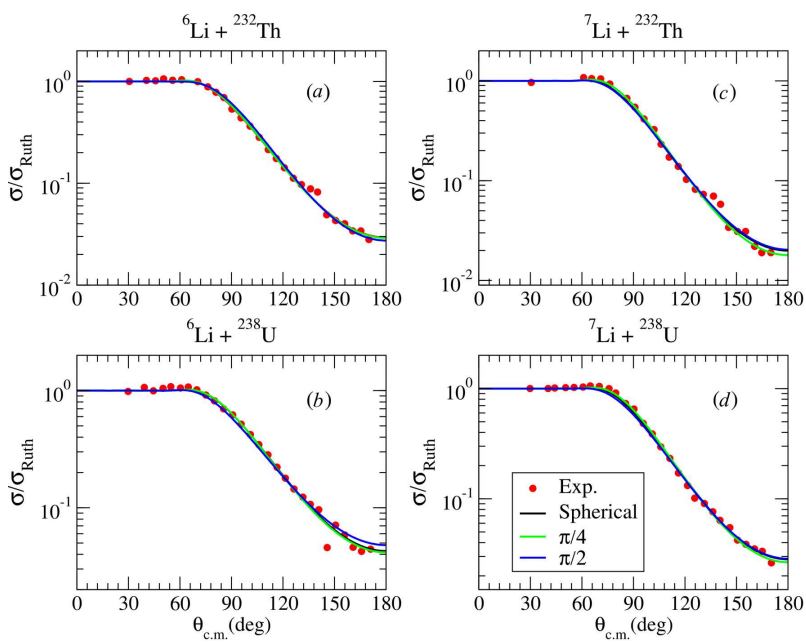

Fig. 2. The calculated cross-sections of the elastic scattering reactions for actinide targets with spherical and deformed surface diffuseness parameters at $\theta=\pi / 4$ and $\theta=\pi / 2$. ${ }^{6} \mathrm{Li}+{ }^{232} \mathrm{Th}$ at $37.2 \mathrm{MeV}(a),{ }^{6} \mathrm{Li}+{ }^{238} \mathrm{U}$ at $37.2 \mathrm{MeV}(b)$, ${ }^{7} \mathrm{Li}+{ }^{232} \mathrm{Th}$ at $37.2 \mathrm{MeV}(c),{ }^{7} \mathrm{Li}+{ }^{238} \mathrm{U}$ at $37.2 \mathrm{MeV}(d)$. The experimental data are taken from Ref. [20]

${ }^{18} \mathrm{O}+{ }^{174} \mathrm{Yb}$, and ${ }^{19} \mathrm{~F}+{ }^{159} \mathrm{~Tb}$ reactions with the lanthanide targets. The elastic scattering cross-sections calculated for spherical and deformed surface diffuseness parameters at two different orientation angles have been presented with the experimental data in Fig. 3. For ${ }^{12} \mathrm{C}+{ }^{152} \mathrm{Sm}$ reaction, it has been observed that the results of the spherical and $\theta=\pi / 4$ cases are 
Table 2. The $W_{0}$ (in $\left.\mathrm{MeV}\right), r_{w}$ (in $\left.\mathrm{fm}\right), a_{w}$ (in $\left.\mathrm{fm}\right), \sigma$ (in $\mathrm{mb}$ ) and $\frac{\chi^{2}}{N}$ obtained from the theoretical analysis with spherical diffuseness parameter, deformed diffuseness parameter, and different orientations for the elastic scattering reactions with actinide and lanthanide targets

\begin{tabular}{|c|c|c|c|c|c|c|c|}
\hline Reaction & $E_{\text {lab }}$ & Orientation & $W_{0}$ & $r_{w}$ & $a_{w}$ & $\sigma$ & $\frac{\chi^{2}}{N}$ \\
\hline \multirow[t]{3}{*}{${ }^{6} \mathrm{Li}+{ }^{232} \mathrm{Th}$} & 37.2 & Spherical & 20.0 & 1.270 & 0.72 & 829 & 0.42 \\
\hline & & $\pi / 4$ & 20.0 & 1.267 & 0.71 & 809 & 0.43 \\
\hline & & $\pi / 2$ & 23.0 & 1.161 & 0.88 & 813 & 0.59 \\
\hline \multirow{3}{*}{${ }^{6} \mathrm{Li}+{ }^{238} \mathrm{U}$} & 37.2 & Spherical & 24.0 & 1.266 & 0.70 & 773 & 1.54 \\
\hline & & $\pi / 4$ & 23.0 & 1.266 & 0.70 & 765 & 1.42 \\
\hline & & $\pi / 2$ & 29.0 & 1.280 & 0.70 & 847 & 2.39 \\
\hline \multirow[t]{3}{*}{${ }^{7} \mathrm{Li}+{ }^{232} \mathrm{Th}$} & 37.2 & Spherical & 25.0 & 1.254 & 0.71 & 887 & 1.10 \\
\hline & & $\pi / 4$ & 20.0 & 1.247 & 0.71 & 820 & 1.00 \\
\hline & & $\pi / 2$ & 23.5 & 1.247 & 0.75 & 909 & 1.23 \\
\hline \multirow[t]{3}{*}{${ }^{7} \mathrm{Li}+{ }^{238} \mathrm{U}$} & 37.2 & Spherical & 24.0 & 1.259 & 0.70 & 815 & 0.30 \\
\hline & & $\pi / 4$ & 20.0 & 1.260 & 0.70 & 779 & 0.30 \\
\hline & & $\pi / 2$ & 22.0 & 1.260 & 0.76 & 882 & 0.42 \\
\hline \multirow[t]{3}{*}{${ }^{12} \mathrm{C}+{ }^{152} \mathrm{Sm}$} & 63.2 & Spherical & 9.00 & 1.350 & 0.86 & 1472 & 5.29 \\
\hline & & $\pi / 4$ & 10.0 & 1.350 & 0.86 & 1506 & 8.04 \\
\hline & & $\pi / 2$ & 8.00 & 1.390 & 0.86 & 1533 & 26.9 \\
\hline \multirow[t]{3}{*}{${ }^{14} \mathrm{~N}+{ }^{159} \mathrm{~Tb}$} & 236.0 & Spherical & 34.0 & 1.300 & 0.50 & 3232 & 49.7 \\
\hline & & $\pi / 4$ & 34.0 & 1.290 & 0.55 & 3289 & 35.4 \\
\hline & & $\pi / 2$ & 9.00 & 1.270 & 0.50 & 2650 & 5.42 \\
\hline \multirow[t]{3}{*}{${ }^{18} \mathrm{O}+{ }^{174} \mathrm{Yb}$} & 83.0 & Spherical & 10.0 & 1.250 & 0.55 & 617 & 12.6 \\
\hline & & $\pi / 4$ & 10.0 & 1.250 & 0.55 & 627 & 14.6 \\
\hline & & $\pi / 2$ & 16.0 & 1.250 & 0.55 & 551 & 0.25 \\
\hline \multirow[t]{3}{*}{${ }^{19} \mathrm{~F}+{ }^{159} \mathrm{~Tb}$} & 98.0 & Spherical & 19.5 & 1.250 & 0.55 & 1017 & 0.25 \\
\hline & & $\pi / 4$ & 24.0 & 1.250 & 0.55 & 1029 & 0.19 \\
\hline & & $\pi / 2$ & 32.0 & 1.250 & 0.55 & 1043 & 0.50 \\
\hline
\end{tabular}

in good agreement with each other and the experimental data. In addition, they are better than the results of $\theta=\pi / 2$. For ${ }^{14} \mathrm{~N}+{ }^{159} \mathrm{~Tb}$ reaction, it has been seen that the results of the spherical and $\theta=\pi / 4$ cases are in accordance with each other. However, the results of $\theta=\pi / 2$ are better compatible with the data than the results of the spherical and $\theta=\pi / 4$ cases. For ${ }^{18} \mathrm{O}+{ }^{174} \mathrm{Yb}$ reaction, it has been noticed that the results of the spherical and $\theta=\pi / 4$ cases are in good agreement with each other, but not with the experimental data. On the one hand, the results of $\theta=\pi / 2$ are in better agreement with the data than the results of the spherical and $\theta=\pi / 4$ cases. For ${ }^{19} \mathrm{~F}+{ }^{159} \mathrm{~Tb}$ reaction, it has been observed that the results of $\theta=\pi / 2$ cannot well define the Fresnel diffraction which is seen in the experimental data, but can describe the other parts of experimental data well. Additionally, the results of $\theta=\pi / 4$ explain slightly better the Fresnel diffraction than the results of $\theta=\pi / 2$. Finally, the results of the spherical case are in good agreement with the Fresnel diffraction, and are better than the results of $\theta=\pi / 4$ and $\theta=\pi / 2$.

The distance-dependent changes of imaginary parts of nuclear potentials for the spherical and deformed surface diffuseness parameters of the elastic scattering reactions with actinide and lanthanide targets have been also shown in Fig. 4. In this respect, the imaginary parts of the nuclear potentials have been assumed as Woods-Saxon potential whose parameters 


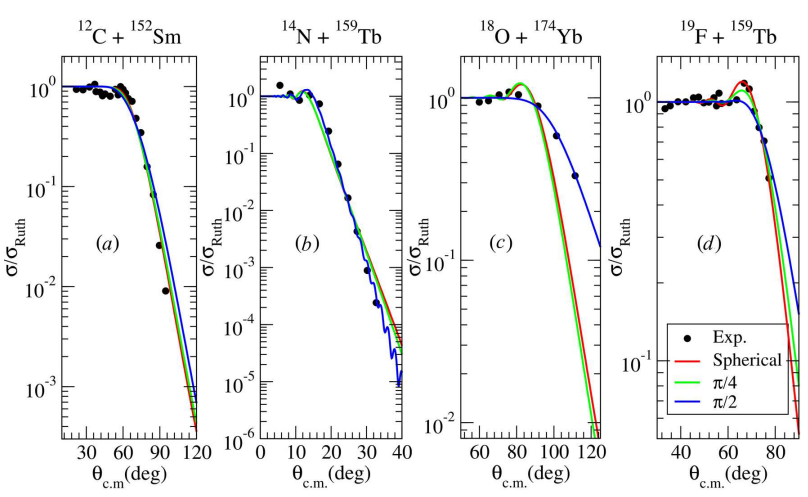

Fig. 3. Same as Fig. 2, but for ${ }^{12} \mathrm{C}+{ }^{152} \mathrm{Sm}$ reaction at $63.2 \mathrm{MeV}(a),{ }^{14} \mathrm{~N}+{ }^{159} \mathrm{~Tb}$ reaction at $236 \mathrm{MeV}(b)$, ${ }^{18} \mathrm{O}+{ }^{174} \mathrm{Yb}$ reaction at $83.0 \mathrm{MeV}(c)$, and ${ }^{19} \mathrm{~F}+{ }^{159} \mathrm{~Tb}$ reaction at $98.0 \mathrm{MeV}(d)$. The experimental data are taken from Refs. [21-24]

have been investigated at 0.1 and 0.01 step intervals in order to achieve good agreement of the theoretical results with the experimental data. The values determined have been listed in Table 2. It is observed that the potential depth and tailing for ${ }^{6} \mathrm{Li}+{ }^{232} \mathrm{Th}$ and ${ }^{6} \mathrm{Li}+{ }^{238} \mathrm{U}$ reactions have been almost the same for the spherical and $\theta=\pi / 4$ situations, whereas both depth and tailing of the imaginary potential at $\theta=\pi / 2$ are different compared to the spherical and $\theta=\pi / 4$ situations. Although the potential depths of ${ }^{7} \mathrm{Li}+{ }^{232} \mathrm{Th}$ and ${ }^{7} \mathrm{Li}+{ }^{238} \mathrm{U}$ reactions differ in three situations, their potential tailing are close to each other. In lanthanide target reactions, the potential depths of ${ }^{12} \mathrm{C}+{ }^{152} \mathrm{Sm}$ reaction are different from each other in three situations, but their potential tailing are very close to each other. Both potential depths and tailing for spherical and $\theta=\pi / 4$ situations of ${ }^{14} \mathrm{~N}+{ }^{159} \mathrm{~Tb}$ and ${ }^{18} \mathrm{O}+{ }^{174} \mathrm{Yb}$ reactions are almost the same. For ${ }^{19} \mathrm{~F}+{ }^{159} \mathrm{~Tb}$ reaction, the potential depths and the potential tailing are different from each other in three situations.

We have given the elastic scattering cross-sections for all the reactions and orientations examined in our study in Table 2. For actinite targets, the behavior of cross-sections has been noticed to be similar to each other. Except for ${ }^{6} \mathrm{Li}+{ }^{232} \mathrm{Th}$ reaction, the largest cross-section values have been obtained in the deformed surface diffuseness case at $\theta=\pi / 2$. We have observed that the elastic scattering cross-sections with deformed surface diffuseness of lanthanide target reactions have been observed to change. The largest cross-section has been obtained

ISSN 2071-0194. Ukr. J. Phys. 2021. Vol. 66, No. 2
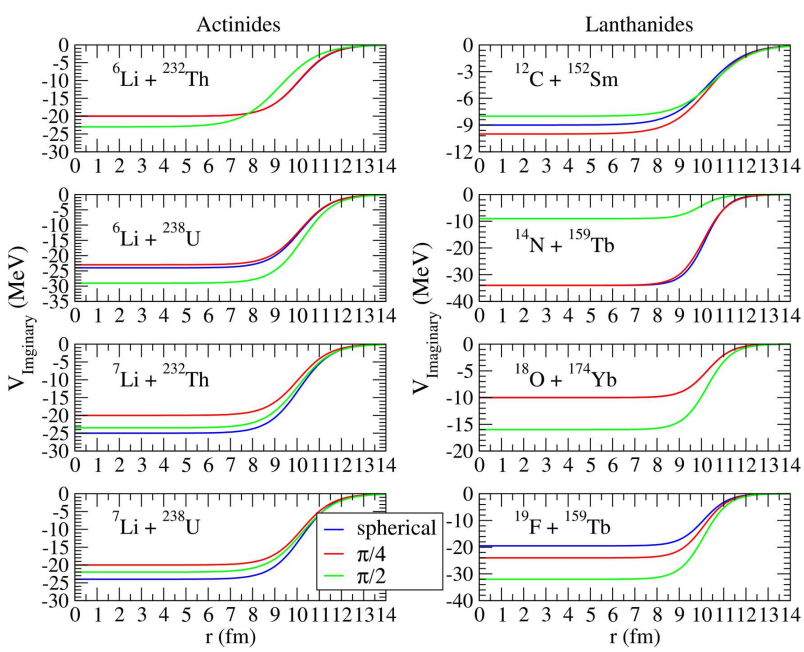

Fig. 4. Distance-dependent changes of the imaginary parts of nuclear potentials for the spherical diffuseness parameter, deformed diffuseness parameter, and different orientations of actinide and lanthanide target reactions
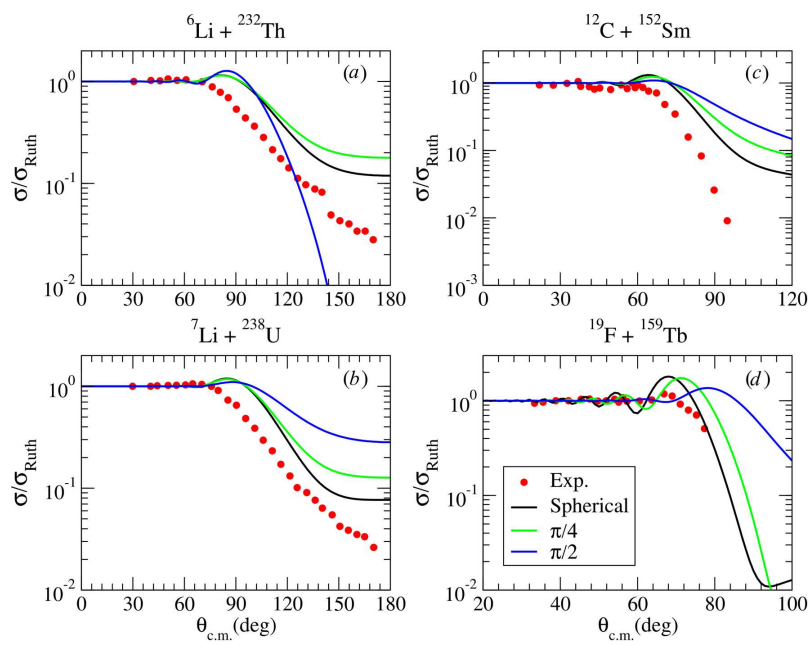

Fig. 5. The elastic scattering cross-sections calculated for $a_{w}=0.1 \mathrm{fm}$ for the spherical, $\theta=\pi / 4$, and $\theta=\pi / 2$ situations. ${ }^{6} \mathrm{Li}+{ }^{232} \mathrm{Th}(a),{ }^{7} \mathrm{Li}+{ }^{238} \mathrm{U}(b),{ }^{12} \mathrm{C}+{ }^{152} \mathrm{Sm}(c)$, ${ }^{19} \mathrm{~F}+{ }^{159} \mathrm{~Tb}(d)$

for $\theta=\pi / 2$ in ${ }^{12} \mathrm{C}+{ }^{152} \mathrm{Sm}$ and ${ }^{19} \mathrm{~F}+{ }^{159} \mathrm{~Tb}$ reactions, and for $\theta=\pi / 4$ in ${ }^{14} \mathrm{~N}+{ }^{159} \mathrm{~Tb}$ and ${ }^{18} \mathrm{O}+{ }^{174} \mathrm{Yb}$ reactions. As a result, it can be said that the deformed surface diffuseness parameter has an effect on the elastic scattering cross-sections. In order to determine whether the effect of the deformed surface diffuseness parameter is large or not, we have performed some new calculations with a very small diffuseness parameter $\left(a_{w}=0.1 \mathrm{fm}\right)$ for ${ }^{6} \mathrm{Li}+{ }^{232} \mathrm{Th},{ }^{7} \mathrm{Li}+{ }^{238} \mathrm{U}$, 
${ }^{12} \mathrm{C}+{ }^{152} \mathrm{Sm}$ and ${ }^{19} \mathrm{~F}+{ }^{159} \mathrm{~Tb}$ reactions. The $W_{0}$ and $r_{w}$ values are the same as in Table 2 . We have presented the theoretical results as compared with the experimental results in Fig. 5. We have observed that the spherical and $\pi / 4$ results for these reactions differ from each other, but not as much as the $\pi / 2$ results. So, the $\pi / 2$ situation has given much different results than the spherical and $\pi / 4$ ones. Thus, we can conclude that the results with spherical and deformed diffuseness parameter are different from each other for the same diffuseness parameter $\left(a_{w}=0.1 \mathrm{fm}\right)$, but the more obvious difference has been observed in different orientation states.

\section{Summary and Conclusions}

In the present study, we have investigated the effect of a deformed surface diffusion parameter on the cross-sections of the elastic scattering reactions with actinide and lanthanide targets. In this respect, we have calculated the elastic scattering cross-sections of all the reactions for the spherical, $\theta=\pi / 4$, and $\theta=\pi / 2$ states. We have observed that the theoretical results agree with the behavior of the experimental data well. Additionally, we have realized that the deformed surface diffuseness parameter has an effect on the elastic scattering cross-sections. Consequently, we can conclude that both spherical and deformed Broglie-Winther potentials can be used in the theoretical analysis of the elastic scattering reactions with actinide and lanthanide targets.

The author would like to thank the anonymous referee for valuable comments.

1. R.D. Woods, D.S. Saxon. Diffuse surface optical model for nucleon-nuclei scattering. Phys. Rev. 95, 577 (1954).

2. J. Błocki, J. Randrup, W.J. Świątecki, C.F. Tsang. Proximity forces. Ann.Phys. (NY) 105, 427 (1977).

3. G.R. Satchler, W.G. Love. Folding model potentials from realistic interactions for heavy-ion scattering. Phys. Rep. 55, 183 (1979).

4. M. Aygun. Effects of proximity potentials on the crosssections of ${ }^{6,8} \mathrm{He}+{ }^{65} \mathrm{Cu}$ halo fusion reactions. Ukr. J. Phys. 64, 363 (2019).

5. M. Aygun. Analysis with SDHO and RMF density distributions of elastic scattering cross-sections of oxygen isotopes $\left({ }^{16-18} \mathrm{O}\right)$ by various target nuclei. Int. J. Mod. Phys. E 27, 1850055 (2018)

6. M. Aygun. A comprehensive analysis of ${ }^{9} \mathrm{Li}+{ }^{70} \mathrm{Zn} \mathrm{fu}-$ sion cross-section by using proximity potentials, temper- ature dependent density distributions and nuclear potentials. Rev. Mex. Fis. 65, 573 (2019).

7. M. Aygun. A comparison of proximity potentials in the analysis of heavy-ion elastic cross-sections. Ukr. J. Phys. 63, 881 (2018).

8. M. Aygun. Alternative Potentials Analyzing the Scattering cross-sections of $7,9,10,11,12,14 \mathrm{Be}$ Isotopes from a ${ }^{12} \mathrm{C}$ target: Proximity potentials. J. Korean Phys. Soc. 73, 1 (2018).

9. M. Aygun, Z. Aygun. A study on ${ }^{7} \mathrm{Li}+{ }^{120} \mathrm{Sn}$ quasi-elastic scattering. Turk. J. Phys. 40, 12 (2016).

10. M. Aygun. The application of some nuclear potentials for quasielastic scattering data of the ${ }^{11} \mathrm{Li}+{ }^{28} \mathrm{Si}$ reaction and its consequences. Turk. J. Phys. 42, 302 (2018).

11. M. Aygun. Comparative analysis of proximity potentials to describe scattering of ${ }^{13} \mathrm{C}$ projectile off ${ }^{12} \mathrm{C},{ }^{16} \mathrm{O},{ }^{28} \mathrm{Si}$ and ${ }^{208} \mathrm{~Pb}$ nuclei. Rev. Mex. Fis. E 64, 149 (2018).

12. S.A. Alavi, V. Dehghani. Investigation of heavy-ion fusion with deformed surface diffuseness: Actinide and lanthanide targets. Phys. Rev. C 95, 054602 (2017).

13. S.A. Alavi, V. Dehghani. Alpha-induced reaction crosssection for $\mathrm{Sm}, \mathrm{U}, \mathrm{Np}$ targets: influence of hexadecapole deformation and deformed surface diffuseness. Chin. Phys. C 41, 064104 (2017).

14. S. Dahmardeh, S.A. Alavi, V. Dehghani. Influence of deformed surface diffuseness on alpha decay half-lives of actinides and lanthanides. Nucl. Phys. A 963, 68 (2017).

15. G.R. Satchler. Direct Nuclear Reactions (Oxford Univ. Press, 1983).

16. I.J. Thompson. Coupled reaction channels calculations in nuclear physics. Computer Phys. Rep. 7, 167 (1988).

17. W. Reisdorf. Heavy-ion reactions close to the Coulomb barrier. J. Phys. G, Nucl. Part. Phys. 20, 1297 (1994).

18. G.L. Zhang, Y.J. Yao, M.F. Guo, M. Pan, G.X. Zhang, X.X. Liu. Comparative studies for different proximity potentials applied to large cluster radioactivity of nuclei. Nucl. Phys. A 951, 86 (2016).

19. P. Möller, A.J. Sierk, T. Ichikawa, H. Sagawac. Nuclear ground-state masses and deformations: FRDM (2012). At. Data Nucl. Data Tables 109-110, 1 (2016).

20. H. Freiesleben, G.T. Rizzo, J.R. Huizenga. ${ }^{6} \mathrm{Li}$ and ${ }^{7} \mathrm{Li}$ induced fission of ${ }^{232} \mathrm{Th}$ and ${ }^{238} \mathrm{U}$. Phys. Rev. C 12, 42 (1975).

21. K. Zhao, X. Lu, Y. Cheng, Q. Li, M. Li, Z. Li, J. Guo, S. Li, Q. Zhang, X. Song, C. Jiang. Comparison of the elastic and inelastic scatterings between ${ }^{152} \mathrm{Sm}+{ }^{12} \mathrm{C}$ and ${ }^{148} \mathrm{Nd}+{ }^{16}$ 0. Z. Phys. A 348, 95 (1994).

22. G.J. Balster, P.C.N. Crouzen, P.B. Goldhoorn, R.H. Siemssen, H.W. Wilschut. Systematics of the ${ }^{14} \mathrm{~N}+{ }^{159} \mathrm{~Tb}$ reaction between 6 and $22 \mathrm{MeV} / \mathrm{u}$ : (I). Inclusive data. Nucl. Phys. A 468, 93 (1987).

23. P.K. Sahu, R.K. Choudhury, D.C. Biswas, B.K. Nayak. Evidence for correlated pair transfer of valence nucleons in multiparticle stripping channels in the ${ }^{18} \mathrm{O}+{ }^{174} \mathrm{Yb}$ reaction. Phys. Rev. C 64, 014609 (2001).

ISSN 2071-0194. Ukr. J. Phys. 2021. Vol. 66, No. 2 
24. A. Kumar, R.Tripathi, S. Sodaye, K.Sudarshan, P.K. Pujari. Angular distributions and cross-sections of projectilelike fragments in the ${ }^{19} \mathrm{~F}+{ }^{159} \mathrm{~Tb}$ reaction. Eur. Phys. J. A 49, 3 (2013).

\section{М. Айгун}

\section{ВПЛИВ ДИФУЗНОСТІ ДЕФОРМОВАНОЇ}

ПОВЕРХНІ НА РЕАКЦІЇ ПРУЖНОГО РОЗСІЯННЯ

НА МІШЕНЯХ $З$ АКТИНІДАМИ І ЛАНТАНІДАМИ

Досліджено вплив дифузності деформованої поверхні на реакції пружного розсіювання на мішенях 3 актинідами і лантанідами. Розраховано перетини пружного розсіяння у припущенні сферичної структури і як сферичної, так і деформованої структури ядер мішені. Розрахунки виконано з використанням сферичного і деформованого потенціалів Бройля-Вінтера для дійсного потенціалу та потенціалу Вудса-Саксона для уявного потенціалу в рамках оптичної моделі. Вплив дифузності деформованої поверхні розглянуто для двох різних кутів орієнтації $\theta=\pi / 4$ i $\theta=\pi / 2$. Проведено порівняння теоретичних результатів між собою і з експериментальними даними.

Ключові слова: дифузність поверхні ядра, деформація, орієнтація, пружне розсіювання, оптична модель. 